\section{Testing vision testing: quantifying the effect of movement on visual acuity measurement}

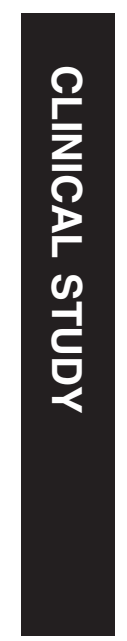

Introduction

Visual acuity (VA) results are fundamental in the detection and management of a range of conditions, therefore it is essential that any variability in measurement is minimised. Studies have documented the test/re-test variability for the assessment of VA fall in the range of $0.01-0.18 \log$ MAR. ${ }^{1-7}$ This variability is attributed, in part, to the inherent variation in visual processing. However, the large disagreement in the range of test/re-test variability, suggests that factors external to visual processing may also contribute to this variability.

One potential source of variability is related to test distance. When charts were developed for use at distances nearer than $6 \mathrm{~m}$, such as 3 and $4 \mathrm{~m}$, it was shown that similar levels of VA were recorded at each distance. ${ }^{8}$ In the complete absence of any ocular defect it could be irrespective of the test distance, as shown by Christoff et al. ${ }^{9}$ However, concern has been expressed that: 'a proportionally greater error could occur if a patient were to lean closer to the chart at $3 \mathrm{~m}$ than at $6 \mathrm{~m}^{\prime} .8$

As LogMAR charts have a consistent progression of optotype size and spacing, adjusting testing distance has an equal impact on each line, allowing precise measurement. While it has been demonstrated that increasing distance between the chart and subject worsens the VA score achieved by a predictable amount, ${ }^{7}$ we found no reports quantifying the amount of patient movement during assessment. Therefore the aim of the present study is to quantify changes in chart/patient separation that may occur during near and distance VA testing, and measured and the movement observed. assumed that VA would be the same to analyse any relationship between the VA
${ }^{1}$ Directorate of Orthoptics and Vision Science, University of Liverpool, Liverpool, UK

${ }^{2}$ Department of Psychological Sciences, University of Liverpool, Liverpool, UK

Correspondence: AR O'Connor, Directorate of Orthoptics and Vision Science, University of Liverpool, Thompson Yates Building, Brownlow Hill, Liverpool L69 3GB, UK Tel: + 4401517945733 ; Fax: + 4401517945781 . E-mail: annaoc@liv.ac.uk

Received: 7 January 2014 Accepted in revised form: 8 September 2014 Published online: 24 October 2014 


\section{Materials and methods}

Ethical approval was granted by the University of Liverpool Ethics Sub-committee. The experiments were performed in accordance with the ethical standards laid down in the Declaration of Helsinki. Informed consent was obtained from the subjects after explanation of the nature and possible consequences of the study.

This study compared VA and subject/VA chart separation. It compared VA score obtained by orthoptists using typical clinical methods to assess VA, where head movements and typical variations of test distance are not controlled, against VA score when head and optotype position were fixed. Differences in resultant VA scores were compared with theoretical predictions, to determine if changes in chart/patient separation caused a variation in VA.

Subjects naïve to vision testing were recruited from within the University of Liverpool and qualified orthoptists were recruited from a number of hospital departments. The aim of using multiple orthoptists and subjects of varying ages, was that the amount of movement recorded, should reflect the amount of movement that occurs in the general adult population. To ensure naïvety, orthoptic students who had been taught VA testing theory and technique were excluded from being subjects.

To determine the sample size required for the present study, Altman's nomogram was used. ${ }^{10}$ As no papers were found that assessed patient movement during testing, papers relating to test/re-test variability were used to determine the population SD. ${ }^{1-7}$ The values of test/re-test variability of children and adults range from 0.01-0.18 logMAR. Using these values to calculate sample size suggested that we would require between 8 and 50 subjects, therefore we aimed to recruit 50 subjects to ensure an appropriate sample.

The recruited orthoptists and subjects were informed that they were participants in a VA test/re-test variability experiment with video monitoring used to monitor protocol compliance, but were not informed that test distance was being measured to avoid influencing test procedures.

Two testing areas were set-up and partitioned, each lit by overhead illumination of $500 \pm 2001 x$, for both near and distance VA tests. We have previously found that this range of illuminance levels ( $\pm 200 \mathrm{~lx}$ ) (Tidbury, Czanner, Newsham, Fiat Lux unpublished) causes no significant difference in VA scores. Two separate testing areas were necessary to avoid the subject or orthoptists being biased in any way at all through observing the strict distance set-up.

Video recording of the assessment was commenced by the recruited orthoptist, using a digital camera mounted on a tripod, positioned to capture a side view of the subject and near vision chart. The orthoptist was provided with an instruction sheet containing the subjects unique ID and a computer generated, randomised testing order (right or left eye, near or distance).

As per the instruction sheet, monocular VA was assessed at $40 \mathrm{~cm}$ using reduced early treatment diabetic retinopathy study (ETDRS) double-sided logMAR charts, and $3 \mathrm{~m}$ 'revised' ETDRS logMAR charts. VA was scored with a slight modification to the letter-by-letter method, ${ }^{5}$ where all mistakes prior to the penultimate line are ignored from the final score. Habitual correction was worn by subjects if necessary, and it was ensured that the same correction was worn during orthoptist assessment and control/research assessment. The orthoptists were instructed to encourage the subjects to attempt optotype identification until five letters were identified incorrectly. The score recorded by the orthoptist is designated the 'orthoptist VA score'. No specific instructions were given other than to follow the protocol and how to start the camera, in an attempt not to bias the clinician, as the aim of this study was to determine what was happening in practice.

When the orthoptist completed the VA assessment and the video recording stopped, the subject was instructed to move through to the researcher room. Masked to the orthoptist VA score, VA was tested by the researcher (LPT) under conditions that controlled for subject movement, through the use of a chin rest and the near VA chart placed on a stand to eliminate chart movement. The near vision chart was at a fixed angle on a stand, adjusted to match $( \pm 2001 x)$ the illuminance at the distance vision chart. Testing order was again randomised, using a data sheet containing the corresponding subject ID. The score recorded is designated the 'control score'. For both the orthoptist and researcher assessment, two variations of the distance VA chart were used. Three variations of the distance ETDRS chart were used (four charts in total) and each side of the near chart was randomly permutated across testing to avoid consecutive use of the same chart/side. The requirement of the subject to read out the entire chart should avoid any practice effect at threshold level.

The video file was imported into a frame-by-frame analysis programme (Carlson GA, DataPoint (Version 0.62), Xannah Applied Science and Engineering, Saint Peters, MO, USA, 2003), which outputs co-ordinate data for each pixel selected. Figure 1 shows a schematic of the captured elements with the $x$ - and $y$-axes illustrated, all measurements were based on relative differences between co-ordinates, with distances measured against the near vision chart, which had a known height of $19 \mathrm{~cm}$. 


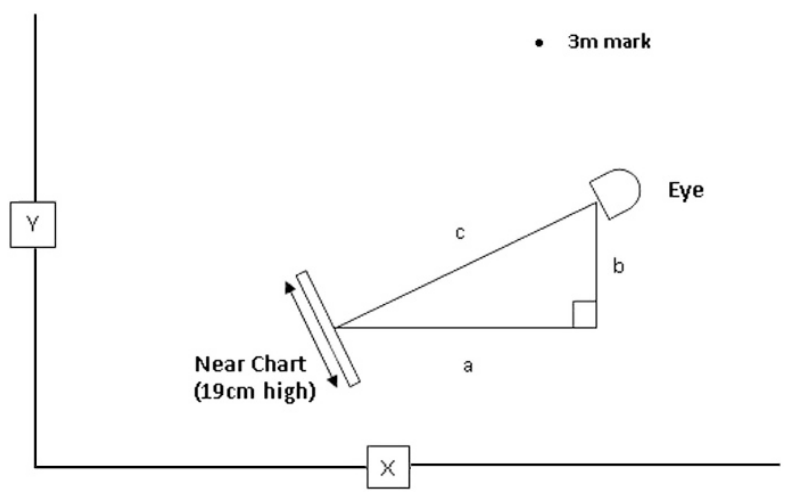

Figure 1 Schematic of a captured video frame. The near chart was used as a ruler, against which all measurements were made. The background during testing was a solid partition divider, with the $3 \mathrm{~m}$ mark attached.

To analyse subject motion during distance vision testing, a fixed marker exactly $3 \mathrm{~m}$ from the distance test chart was placed beyond the subject (Figure 1). Any motion of the subject forward or backward from this point was analysed, the difference between the marker and subject's eye defining the amount of movement. For near vision testing, the co-ordinates of the test chart and the eye of the subject were recorded. The difference ' $c$ ' between the $x$ and $y$ co-ordinates of the centre of the chart and eye ' $a$ and $b$ ', were used to construct a triangle to determine the change in distance between the chart and the subject eye.

On the assumption that VA would be best when separation between the eye and the VA chart was smallest, the subject/chart separation at this point was determined for each subject. Another assumption would be that the distance at the end of the test, when the subject is reaching threshold, would be the most indicative of VA level; therefore the final testing distance was also extracted from the data for analysis. To preserve the magnitude of the testing distances for each point when averaging the distances, the results were separated into those subjects who moved towards the chart and those that moved away (otherwise those who moved away, would remove the effect of those who moved towards, and vice versa). The closest and final proximity of the range of distances moved for each subject was used to calculate the expected change in VA. This produced the 'predicted VA score'.

The design rule that a reduction of $80 \%$ of the distance between chart and subject reduces angular size of the optotypes by $25 \%, 11$ allows us to calculate the effect of changing the viewing distance of any VA chart. Table 1 demonstrates the effect on VA score for a 6, 4, 3, and $0.40 \mathrm{~m}$ chart. To calculate the effect of increasing separation, the design rule was inversed. These calculations were verified with figures given in the design paper and by comparison to other studies, which used increasing patient/chart distance, to artificially reduce VA by a predetermined number of logMAR lines. ${ }^{7,11}$

The data collected for each eye consisted of the 'orthoptist score' (written down by the orthoptists) and 'control score' (recorded by LPT with fixed distances) for both near and distance VA with the addition of the 'video score'. The video score was based on analysis of the video recording of the subject assessment with the orthoptist re-scored by the researcher (LPT), to ensure that a consistent scoring method was used and any recording error negated.

\section{Statistical methods}

The normality of the data was assessed using the Kolmogorov-Smirnov test alongside visual inspection of the histogram. Mean \pm SD are presented if the data is normally distributed and if median \pm interquartile range is non-normal. Statistical analyses tested for differences between video and control score, predicted and control score, and video and predicted score. Friedman's ANOVA was used with post-hoc testing to examine individual differences. Differences in VA scores were represented using Bland-Altman plots. ${ }^{10}$

\section{Results}

\section{Subjects}

Sixteen orthoptists and 51 subjects, aged 18-73 years, were recruited from staff and students at the University of Liverpool, with each orthoptist testing three to five subjects each. Each eye of the subject was used as an 'individual', resulting in 102 recordings for both near and distance. The movement analysis provided 97 sets of data for distance VA testing and 85 for near, with missing cases resultant of an obstructed subject view due to the orthoptists blocking the camera. List-wise deletion of missing points was employed, as missing data due to non-observation of subject movement is random and not likely to affect the movement data that was observed. The amount of movement when testing either eye of the same person was not constant, with non-normal distribution.

\section{Comparison of VA scores with and without movement limited}

The control score is statistically significantly better than the video score $(P<0.001)$. However there is no statistically significant difference between the orthoptist 
Table 1 Predicted effect on visual acuity score based on a reduction of $(-)$ or increase in $(+)$, distance between patient and chart for each $\log M A R$ chart distance calibration

\begin{tabular}{|c|c|c|c|c|c|c|c|c|}
\hline \multirow{3}{*}{$\begin{array}{l}\text { Amount } \\
\text { logMAR VA } \\
\text { score would } \\
\text { change }\end{array}$} & \multicolumn{8}{|c|}{$\begin{array}{c}\text { Predicted effect on visual acuity score based on distance } \\
\text { changes in table }(\mathrm{cm})\end{array}$} \\
\hline & \multicolumn{2}{|c|}{$0.40 \mathrm{~m}$ chart } & \multicolumn{2}{|c|}{$3 \mathrm{~m}$ chart } & \multicolumn{2}{|c|}{$4 m$ chart } & \multicolumn{2}{|c|}{$6 \mathrm{~m}$ chart } \\
\hline & - & + & - & + & - & + & - & + \\
\hline 0.02 & 2 & 2 & 15 & 14 & 21 & 20 & 31 & 28 \\
\hline 0.04 & 4 & 4 & 28 & 29 & 38 & 40 & 57 & 58 \\
\hline 0.06 & 6 & 6 & 40 & 45 & 53 & 60 & 80 & 90 \\
\hline 0.08 & 7 & 8 & 51 & 61 & 68 & 80 & 100 & 122 \\
\hline 0.10 & 8 & 10 & 60 & 78 & 82 & 105 & 123 & 155 \\
\hline 0.20 & 15 & 24 & 110 & 177 & 148 & 234 & 220 & 350 \\
\hline 0.30 & 20 & 40 & 150 & 300 & 200 & 400 & 294 & 587 \\
\hline
\end{tabular}

Moving the $40 \mathrm{~cm}$ chart to $32 \mathrm{~cm}$ would increase VA, theoretically by $0.1 \log$ MAR (one line), and moving it to $50 \mathrm{~cm}$ would reduce it by $0.1 \log$ MAR. A $2 \mathrm{~cm}$ difference would change VA by one letter. At $6 \mathrm{~m}$, the target could move around $33 \mathrm{~cm}$ before a difference of one letter was made and well over $1 \mathrm{~m}$ before a line difference would be made.

and video score $(P>0.3)$. The median scores are shown in Table 2.

The level of agreement was evaluated using BlandAltman (Figure 2). ${ }^{10}$ On average, good agreement is shown between video vs orthoptist VA, with the mean bias being $<0.03$ in all cases. However, the video VA score tends to give a higher (worse) score than the control score.

\section{Relationship between the movement and VA scores}

The amount of movement of the group as a whole is shown in Table 3. When calculating the average amount of movement for the entire group, increases in testing distance would negate any measurement of a decrease in test distance and vice versa. To avoid losing any effect due to this, subjects were separated into those who reduced the testing distance during assessment and those who increased testing distance during testing. These were further divided based on the two possible points that best VA may have been recorded, the closest the subject was to the chart during testing, and the separation when threshold VA were reached.

There are statistically significant differences $(P<0.05)$ between the VA scores achieved in the control condition and the score achieved on the video recording (Table 3 ). To determine if this is attributable to the movement that occurred during testing, the predicted VA score was calculated. Table 4 shows the predicted and video scores for the separations described in Table 3.

The analysis shows that for increased separation (movement away from correct test distance) the expectation of a worsening of VA was confirmed $(P<0.05)$. However, for near testing, a closer testing
Table 2 VA LogMAR score characteristics. (interquartile range $=(\mathrm{IQR}))$

\begin{tabular}{lcr}
\hline & $\begin{array}{c}\text { Near VA, } \\
\mathrm{n}=102\end{array}$ & \multicolumn{1}{c}{$\begin{array}{c}\text { Distance VA, } \\
\mathrm{n}=102\end{array}$} \\
\hline Control score median (IQR) & $-0.100(0.16)$ & $-0.100(0.16)$ \\
Video score median (IQR) & $-0.030(0.20)$ & $-0.080(0.18)$ \\
Orthoptist score median (IQR) & $0.000(0.23)$ & $0.000(0.10)$ \\
\hline
\end{tabular}

distance did not demonstrate an improvement in VA, rather the opposite, where reduced separation during near VA testing resulted in a worsening of VA.

Subjects who moved the most were selected for further analysis. The closest point of these subjects (testing distance reduced $\geq 8 \mathrm{~cm}$ ) would predict a one logMAR line difference on the near vision test. However, of the five who moved towards the chart, three had worsened $\mathrm{VA}$, one improved by a line, and one maintained the same VA as in the control condition.

Consideration was also given to the effect of age on movement amount, as subjects with any uncorrected presbyopia or reading glasses designed for use at distances $\geq 40 \mathrm{~cm}$, may have been inclined to move more. Analysis shows that there is no significant difference in the amount of movement between those aged $<45$ and those aged $\geq 45$ ( $n=9$, Mann-Whitney, $P=0.75$ ).

\section{Discussion}

The observed effect of movement overall for the subjects in this study shows a relatively small impact on VA scores as predicted by movement, the magnitude of which is within the one LogMAR line $95 \%$ confidence interval required to detect a real change. ${ }^{12}$ For the purposes of research, this suggests that movement may not be a crucial factor when determining change of VA for a group of subjects. For clinical applications, however, the group difference is not as important, and we have demonstrated large changes in test distances for individual subjects.

Minimising variations in the viewing distance will improve VA test accuracy. The findings from this study demonstrate that movement does occur during VA testing, resulting in a change in test chart/patient distance of up to $42 \%$ of the specified testing distance. This large change was a result of incorrect set-up, due to the distance cord not being fully extended. Allowing movement during VA testing resulted in a different VA score compared with a fixed viewing distance, with increases up to $24 \mathrm{~cm}$ and decreases up to $17 \mathrm{~cm}$ found for distance VA testing. During near vision testing, $44 \%$ of the cases analysed were at least $4 \mathrm{~cm}$ away from specified test distance, equating to a change of $10 \%$ of the test distance. The use of co-operative adult patients 

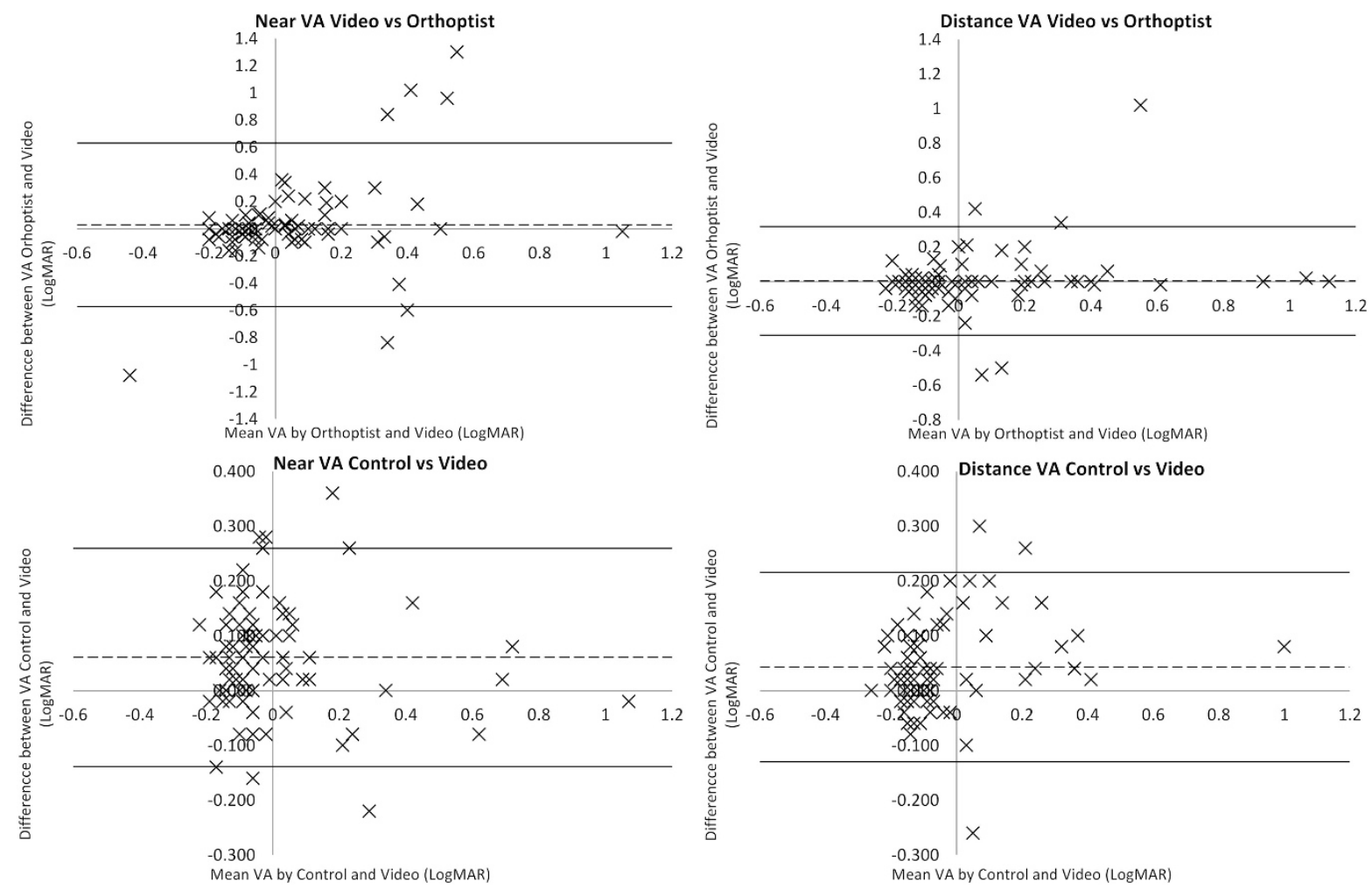

Figure 2 Bland-Altman level of agreement. Dashed lines represent the mean bias. Solid lines above and below the $x$-axis show $95 \%$ confidence interval.

Table 3 Description of the overall range of movement of the subjects, separated into those who increased and those who reduced testing distances during assessment

\begin{tabular}{|c|c|c|c|c|}
\hline \multirow{3}{*}{$\begin{array}{l}\text { Range of movement }(\mathrm{cm}) \\
\text { Point analysed }\end{array}$} & \multirow{2}{*}{\multicolumn{2}{|c|}{$\begin{array}{c}\text { Near VA testing } \\
\begin{array}{c}-12 \text { to }+9 \\
\mathrm{n}=84\end{array}\end{array}$}} & \multirow{2}{*}{\multicolumn{2}{|c|}{$\begin{array}{c}\text { Distance VA testing } \\
\begin{array}{c}-17 \text { to }+24 \\
\mathrm{n}=97\end{array}\end{array}$}} \\
\hline & & & & \\
\hline & Closest & Final & Closest & Final \\
\hline Reduced test distance (cm; median (IQR)) & $\begin{array}{l}-4(3) \\
n=59\end{array}$ & $\begin{array}{l}-3(4) \\
n=34\end{array}$ & $\begin{array}{l}-6(7) \\
n=27\end{array}$ & $\begin{array}{l}-5(8) \\
n=22\end{array}$ \\
\hline Increased test distance (cm; median (IQR)) & $\begin{array}{l}+2(3) \\
n=25\end{array}$ & $\begin{array}{l}+4(3) \\
n=50\end{array}$ & $\begin{array}{l}+9(9) \\
n=70\end{array}$ & $\begin{array}{l}+11(8) \\
n=75\end{array}$ \\
\hline
\end{tabular}

Average subject/chart separations are shown for both the closest point the subject was to the chart, and the final point when the threshold VAs were identified. (interquartile range $=(I Q R)$ ).

aimed to limit the impact of subject variability, to avoid compliance issues, and for the task to be fully understood. However, even with co-operative adult subjects, movement was observed, and in some cases a large amount occurred. These data may represent the minimum variability in movement anticipated when testing children.

As movement and its impact on VA were the primary outcome measures, the experiment was designed to limit other factors that could contribute to test/re-test variability. While the predicted scores indicated the correct direction of VA change in most cases (Table 4), the direction and magnitude was incorrectly predicted for near VA testing, where test distance was reduced. Movement towards the chart predicted an improved VA score, however, a worse score was found. Compared with the predicted score (based on the reduced test distance), the difference is even more marked with a statistically significant reduction of 0.04-0.06 logMAR. There are a few potential reasons for this lack of a direct relationship. While subjects were instructed to wear habitual correction, it is possible that some were undercorrected or not using any correction, and as nine subjects were over the age of 45 years, their movement 
Table 4 Predicted VA scores based on movement and control VA

\begin{tabular}{|c|c|c|c|c|}
\hline & \multicolumn{2}{|c|}{ Near VA LogMAR (median (IQR)) } & \multicolumn{2}{|c|}{ Distance VA LogMAR (median (IQR)) } \\
\hline & Closest & Final & Closest & Final \\
\hline \multicolumn{5}{|c|}{ Reduced test distance } \\
\hline Control & $-0.100(0.160)$ & $-0.100(0.160)$ & $-0.060(0.320)$ & $-0.060(0.340)$ \\
\hline Prediction & $-0.139(0.160)$ & $-0.127(0.172)$ & $-0.084(0.334)$ & $\{-0.078(0.336)$ \\
\hline Video & $-0.080(0.160)$ & $-0.080(0.160)$ & $(-0.080(0.400)$ & $-0.070(0.480)$ \\
\hline \multicolumn{5}{|c|}{ Increased test distance } \\
\hline Control & $-0.120(0.100)$ & $-0.120(0.140)$ & $-0.100(0.100)$ & $-0.100(0.100)$ \\
\hline Prediction & $-0.070(0.144)$ & $-0.058(0.140)$ & $-0.093(0.105)$ & $-0.094(0.105)$ \\
\hline Video & $0.000(0.140)$ & $0.000(0.160)$ & $-0.080(0.160)$ & $-0.080(0.160)$ \\
\hline
\end{tabular}

Parentheses indicate non-significant differences. Grey shading indicates correct direction prediction of VA score based on movement.

towards the chart could have resulted in a worsening score. It would seem counterintuitive that these subjects would choose to move to an area that would degrade their VA, and no statistically significant difference was found to those aged $<45$.

While earlier we determined a clinically relevant difference as $0.1 \log$ MAR this amount does not directly apply to our findings, as this study recognises changes in angular size rather than the control method. Standard scoring systems attribute $0.02 \log$ MAR to each optotype, and a 0.02 difference in VA measures would not be considered to be of clinical or statistical significance. However, this linear approach to scoring is not precise, as the angular size of the optotypes does not vary across the line. The equations used to calculate the score effect are based on the logarithmic decrease in the angular size of the letters on each line, as per the design principles, meaning that a predicted change of 0.02 represents an effect on the entire chart. While this inherent flaw is present in all VA tests and may account for some of the variability in test/re-test, the findings here demonstrate changes in VA beyond those accountable by the test chart limits.

During testing there were multiple anecdotal reports, suggesting an influence of lateral movement or 'wobble' of the near VA chart. Subjects reported to the researcher that they found it easier to read the near chart when mounted on a stand. The ability to resolve fine detail is greatest when the stimuli is stationary, as the spatial scale used when motion is present is coarser, resulting in a degradation of VA. ${ }^{13,14}$ It is likely that when approaching threshold levels of VA, the ability to detect form could be greatly hindered, even by small amounts of lateral motion/wobble of the test chart.

Variations in chart presentation angle can change the level of optotype illumination by 430 lx, which our currently un-submitted work has shown to worsen VA. However, analysis of the video showed that the chart tends to be held at $45^{\circ}$ during testing, maintaining a good level of illuminance.

Other factors such as refractive error or accommodation may influence the VA scores achieved for near. While the effect of any uncorrected refractive error could be exacerbated by reducing or increasing test distance, it would seem counterintuitive for the subject to move to worsen their vision. We did not measure the refraction of the subjects, as our aim was to measure the amount of movement that occurs during vision testing. Analysis demonstrated that those aged $\geq 45$ did not move more than those below this age, suggesting that movement to improve VA due to inappropriate correction for the $40 \mathrm{~cm}$ test distance was not the primary cause of any movement.

For near vision testing, one clinician had near zero measurable deviation from test distance. This clinician, rather than using the cord to only set initial test distance, had the subject hold the cord end next to their eye throughout, whilst maintaining tension of the cord, by gently pulling the chart away from the subject. This appears to allow adaptation to any subject movement, through recognising any change in tension.

Overall, these data suggest that by ensuring that the correct testing distances are used and maintained, variability of VA testing will improve. In addition, for near testing, the placement of the test type on a stand will reduce the effect of 'wobble' and/or the use of the distance setting cord will allow greater control of the test distance. Measures to minimise patient movement would also be beneficial, as would the use of an enlarged copy of the test for the clinician to use for scoring purposes. This will minimise errors in recalling which optotypes were identified, and eliminates creating shadows when tracking responses during near VA testing. Further testing by utilising these approaches will allow differentiation of the impact that chart and patient movement have on test/re-test variability from other possible variables. 


\section{Summary}

\section{What was known before}

- VA charts are designed to facilitate testing at different distances, therefore, movement during testing will impact on the measurement. There is variability in repeated measures of visual acuity, which can be up to 0.15 LogMAR.

\section{What this study adds}

- Quantification of the amount of movement, which occurs during visual acuity testing, provides an evaluation of the contribution of movement to the variation in repeated measures of visual acuity.

\section{Conflict of interest}

The authors declare no conflict of interest.

\section{Acknowledgements}

We would like to thank the volunteer orthoptists who took part in the collection of data for this study.

\section{References}

1 Arditi A, Cagenello R. On the statistical reliability of letter-chart visual acuity measurements. Invest Ophthalmol Vis Sci 1993; 34(1): 120-129.

2 Rosser DA, Laidlaw DAH, Murdoch IE. The development of a "reduced $\log M A R "$ visual acuity chart for use in routine clinical practice. Br J Ophthalmol 2001; 85(4): 432-436.

3 Lim L, Frost NA, Powell RJ, Hewson P. Comparison of the ETDRS logMAR, compact reduced logMar and Snellen charts in routine clinical practice. Eye 2010; 24(4): 673-677.
4 Gouthaman M, Raman RP, Kadambi A, Padmajakumari R, Paul PG, Sharma T. A customised portable LogMAR chart with adjustable chart illumination for use as a mass screening device in the rural population. J Postgrad Med 2005; 51(2): 112-114.

5 Ferris III, FL, Kassoff A, Bresnick GH, Bailey L. New visual acuity charts for clinical research. Am J Ophthalmol 1982; 94(1): 91-96.

6 Laidlaw DAH, Abbott A, Rosser DA. Development of a clinically feasible logMAR alternative to the Snellen chart: Performance of the "compact reduced logMAR" visual acuity chart in amblyopic children. Br J Ophthalmol 2003; 87(10): 1232-1234.

7 Rosser DA, Cousens SN, Murdoch IE, Fitzke FW, Laidlaw DAH. How sensitive to clinical change are ETDRS logMAR visual acuity measurements? Invest Ophthalmol Vis Sci 2003; 44(8): 3278-3281

8 Bryant K, Gay E, Hazell C, Harris S, Hollingsworth T, Moseley $\mathrm{M}$ et al. Visual acuitiy: a comparison of 3 and 6 metre test distances. Br Orthopt J 1991; 48: 21-24.

9 Christoff A, Repka MX, Kaminski BM, Holmes JM. Distance versus near visual acuity in amblyopia. J AAPOS 2011; 15(4): 342-344.

10 Bland JM, Altman DG. Statistical methods for assessing agreement between two methods of clinical measurement. Lancet 1986; 1(8476): 307-310.

11 Bailey IL, Lovie JE. New design principles for visual acuity letter charts. Am J Optom Physiol Opt 1976; 53(11): 740-745.

12 Bailey IL, Lovie-Kitchin JE. Visual acuity testing. From the laboratory to the clinic. Vision Res 2013; 90: 2-9.

13 Chung STL, Bedell HE. Velocity dependence of Vernier and letter acuity for band-pass filtered moving stimuli. Vision Res 2003; 43(6): 669-682.

14 Vallam K, Metha AB. The relationship between temporal phase discrimination ability and the frequency doubling illusion. J Vision 2007; 7(14): 17.1-11. 